\title{
Generative Adaptive Testing with Digit Span Items
}

\author{
John H. Wolfe and Gerald E. Larson
}

Testing Systems Department

Navy Personnel Research and Development Center

San Diego, CA 92152

-.--DRAFT--.-

2 January 1990

The opinions expressed in this paper are those of the authors, are not official and do not necessarily reflect the views of the Navy Department. 


\section{INTRODUCTION}

The digit span test has a long history of use in mental ability tests, and remains one of the subtests in the WISC-R and WAIS intelligence tests (Dempster, 1981; Mishra, Ferguson \& King, 1983). In its conventional form, the examiner reads a sequence of digits aloud to the examinee(s), who must repeat them back in either the original or reverse order. The examiner has to be carefully trained, however, to read the digits at a uniform speed and tone, without grouping them or giving other cues to the examinee. In order to standardize administration, tape recordings or visual displays from computers have sometimes been employed. Computerized administration of digit span tests offers many advantages in reducing possible sources of error in administration and in making it possible to test examinees in groups while providing the necessary interaction. Computerized versions of digit span tests have been reported by Slotnick and Jeger (1985), Wilson (1982, 1987), Beaumont (1985), and Webster, Gouvier and Gross, (1983).

In testing situations where repeated administration is needed, such as monitoring performance under stress or treatment conditions, a generative computerized testing system would be desirable. Such a system would generate a unique set of items for each examinee at each testing occasion.

The feasibility of generative testing depends on being able to generate items of known difficulty. This paper will show how to predict the difficulty of a digit span item from its characteristics, and how to link an item generation method to an adaptive testing system for greater measurement precision.

\section{METHOD}

\section{Instrument:}

The calibration pool contained six items at each of six different digit span lengths: $5,6,7,8,9$, and 10 . The items were constructed by using a random number table. The only editing that was done on these random sequences was to eliminate items with three consecutive repeated digits. The items were computer-administered on an HP Integral computer with a 9 inch flat screen.

In any given item, the digits are presented on the center of the screen, one at a time, at 1-second intervals. Following the last digit, a screen appears telling the subject "Please type the numbers in the order that they were presented. _... - (The number of underscores is the number of digits in the item.) As the examinee enters the digits from the numeric keypad, the underscores are replaced by the digits, and the message appears:

"Press $<\mathrm{B}>$ to go back and correct any mistakes."

When the examinee has typed the final digit, the message appears:

"Press $<$ ENTER $>$ if you are done typing your answer."

The test was preceded by six practice items ranging from five to seven digits in length. Fcedback was given after each practice item. The instructions took approximately 3.5 minutes to administer. The 36 test items required approximately 12.5 minutes. 


\section{Subjects}

The items were administered to 531 male recruits at the Navy Recruit Training Center in San Diego. Each examinee took all 36 items. In addition, the examinees took a working memory test, "Mental Counters" (Larson et. al, 1989).

\section{Calibration and Scoring}

The items were calibrated for two different models: the one-parameter logistic model (Rasch) and the two-parameter logistic model. The computer program MULTILOG (Thissen, 1988) was used to obtain marginal maximum likelihood (MML) estimates of the item parameters and maximum a posteriori (MAP) estimates of the examinees' latent abilities.

\section{Hypotheses}

Three hypotheses were tested in this experiment: (1) The psychometric difficulty of the digit span items are closely related to their length. (2) When a digit is repeated one or more times in the sequence, but not consecutively, interference will make the item harder. This case will be referred to as "repetition." (3) When two adjacent digits are the same the item will be easier because of chunking. This case will be referred to as "duplication."

\section{Analysis}

The obtained item parameters were regressed on the number of digits in the item. The residuals from this regression were correlated with the number of digit duplications and repetitions in the item.

\section{RESULTS}

The major results are summarized in Table 1. The standard errors in the table refer to the errors in the MULTILOG estimation process, not regression. MULTILOG estimates for the Rasch difficulties $b_{i}$ ranged from -1.882 to 3.932 with a mean of 1.112 and a standard deviation of 1.800 . The standard errors from the parameter estimation process were estimated by MULTILOG to range from .108 to .415 , with a root mean square of .197. The common discrimination parameter, $a$, was 1.220 . (The scaling factor of 1.7 does not appear in the model. i.e., the model equation is:

$$
P\left(x_{i, j}\right)=\Psi\left(a\left(\theta_{j}-b_{i}\right),\right.
$$

where $x_{i, j}$ is the response of person $j$ to item $i$ and $\Psi$ is the Logistic function.) 
Table 1

MULTILOG IRT Parameter Estimates for 36 Digit Span Items $(\mathrm{N}=531)$

Correlation with

\begin{tabular}{lcccccc} 
Parameter & Mean & Std. Dev. Minimum & Maximum & 5-10 Digits & 5-9 Digits \\
\hline Rasch Difficulty & 1.112 & 1.800 & -1.882 & 3.932 & .986 & .980 \\
MULTILOG Std. Error & .197 & - & .108 & .415 & - & \\
& & & & & & \\
2-PL Difficulty & .842 & 1.577 & -1.966 & 3.658 & .968 & .973 \\
MULTLOG Std. Error & .303 & - & .104 & 1.027 & - & \\
& & & & & & \\
2-PL Discrimination & 1.430 & .480 & .768 & 2.878 & .736 & .812 \\
MULTLOG Std. Error & .310 & - & .133 & .772 & - & \\
\hline
\end{tabular}

Figure (1) shows the least squares regression of the Rasch difficulty on the number of digits in the item. The correlation is .986 and appears linear. The standard error of estimate around the regression line is 0.305 . Thus $42 \%$ of the variance around the regression line is due to error in the original parameter estimation, leaving a variance of .054 , or $58 \%$ still unaccounted for. If there were homoscedasticity, perfect parameter estimation would imply a correlation between difficulty and number of digits of .992 .

Insert Figure 1 about here.

The correlation of the residual difficulty with number of digit repetitions in the sequence was $.296(\mathrm{p}<.04$ for a 1 -tailed t-test). The correlation of the residual difficulty with the number of digit duplications was -.212 (non-significant, although in the expected direction.) The effect on error of estimate was small: adding digit repetitions reduced the residual error to 291 .

In the two parameter logistic model, MULTILOG estimates of the item discrimination parameters, $a_{j}$ ranged from .768 to 2.878 with a mean of 1.430 . Their standard errors ranged from .133 to .772 with a root mean square of .310 . The errors were considerably larger for the more difficult items; for 9 and 10 digit items the root mean square errors were .387 and .563 , respectively. Again, the scaling factor of 1.7 does not appear in the model.

The two parameter model gave a significantly better fit to the item characteristic curves than the Rasch model. The logarithm of the likelihood ratio for the two models was 34.9 . By the usual chi-squared test with 35 degrees of freedom, the probability for the Rasch model is less than .0005 . Inspection of the item characteristic curves for the two models shows no difference for digit spans of 5,6,7, and 10. At digit spans of 8 and 9 , small differences occur for examinees with abilities greater than 1.5 on the theta scale.

The regression results for the two parameter logistic model are shown in Figures (2) and (3). The number of digits in the item correlated .968 with difficulty and .736 with the 


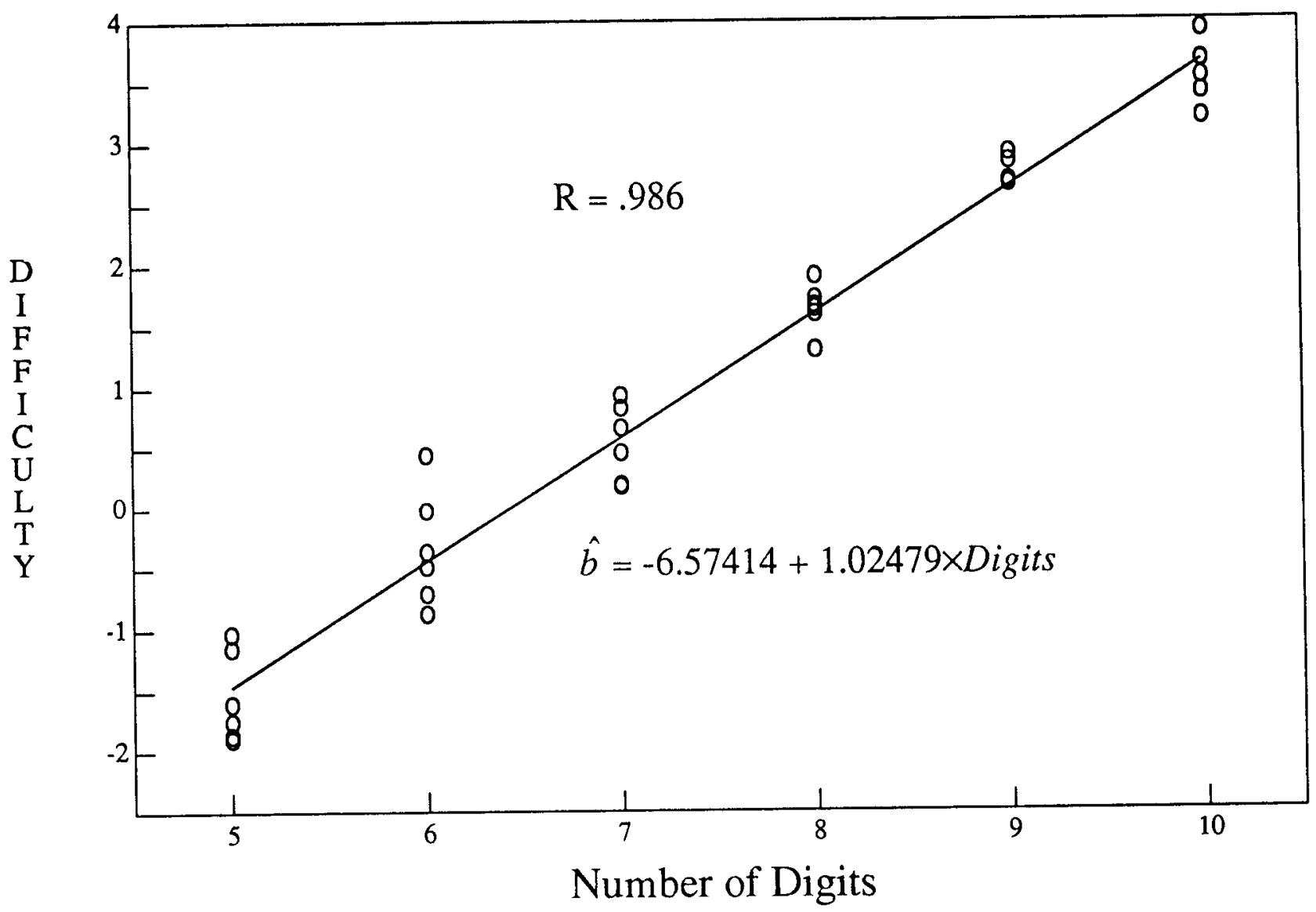

Figure 1. Rasch Item Difficulty for 36 Digit Span Items. 
discrimination parameter. (These figures rise to .973 and .812 if the 10-digit numbers are omitted from the regression analysis.) The standard error around the regression line for the difficulty parameter is .405 . Thus $56 \%$ of the variance around the regression line is due to error in the original parameter estimation, leaving a variance of .072 , or $44 \%$ still unaccounted for. If there were homoscedasticity, perfect parameter estimation would imply a correlation between difficulty and number of digits of .985 .

The regression of the discrimination parameter, $a$, on number of digits is clearly non-linear: the mean $a$ parameter for five digits is greater than for six digits. Thus our use of linear regression for the 2-PL model misses some of the regularity that may be present in the data.

Insert Figure 2 about here.

Insert Figure 3 about here.

\section{SIMULATION OF NON-ADAPTIVE AND ADAPTIVE TESTING}

Simulations were carried out, comparing several versions of non-adaptive and adaptive tests. In the first series of simulations, the item pool was constructed to have the parameters falling on the regression lines shown in the preceding figures. That is, all items of a given length had the same parameters, and these true values were assumed to be known to the adaptive testing procedure without error. Test lengths of 16,24 and 32 items were simulated, using both the Rasch and the 2-PL models.

The adaptive test updated the pseudo-examinee's ability estimate after each item "response" using Owen's bayesian algorithm (Owen, 1975). The next item was selected to have the maximum information for that level of ability. At the end of the test, the examinee's final ability was estimated by the Bayesian modal method rather than Owen's. The items in the pool ranged from 4 digits to 10 digits in length.

The items in the non-adaptive tests ranged from 5 to 8 digits in length. The number 


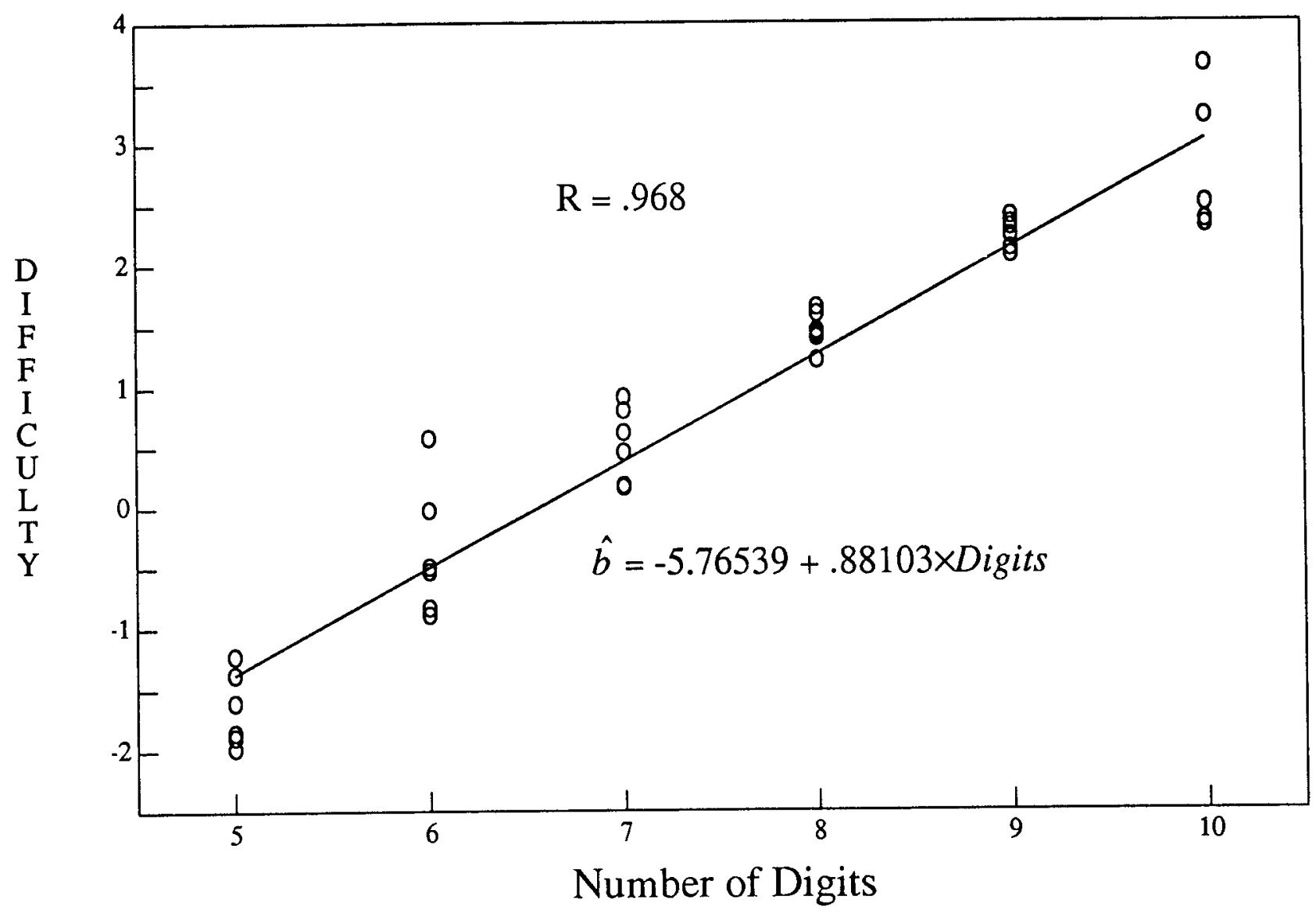

Figure 2. 2-PL Item Difficulty for 36 Digit Span Items. 


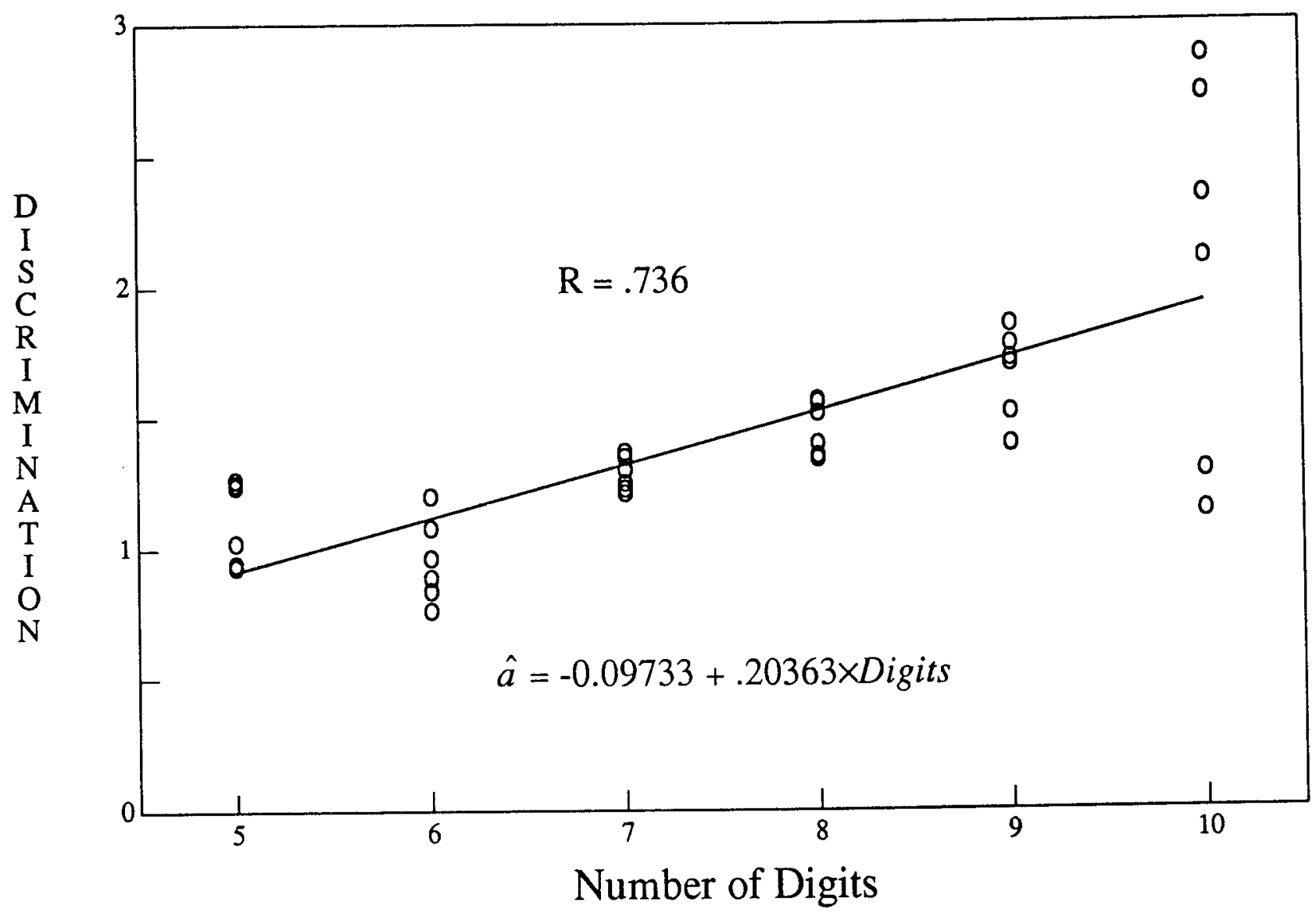

Figure 3. 2-PL Item Discrimination for 36 Digit Span Items. 
of items of each type are shown in Table 2.

Table 2

Non-Adaptive Test Items

Test Length

\begin{tabular}{crrr}
\cline { 2 - 4 } Digits & 16 & 24 & 32 \\
\hline 5 & 3 & 5 & 6 \\
6 & 5 & 7 & 10 \\
7 & 5 & 7 & 10 \\
8 & 3 & 5 & 6 \\
\hline
\end{tabular}

To determine the reliabilities of the tests, normally distributed samples of 155,000 simulated examinees were generated. The results for the Rasch parameters are shown in Table 3 and for the two-parameter logistic model in Table 4. The increases in the reliabilities with test length are in agreement with the Spearman-Brown formula. The second column from the left shows the estimated time for instructions plus testing. The rightmost column shows the difference in reliability between the adaptive and non-adaptive tests of the same length. The results indicate that a gain of about .02 to .05 can be expected in reliability from using adaptive testing. This gain was no more than .006 greater when the 2-PL model was used, a negligible difference. Thus, even though the reliability estimates are higher with the 2-PL parameters for both adaptive and nonadaptive tests, there is little advantage for adaptive tests in preferring the 2 -PL model to the Rasch model.

Table 3

Reliabilities of Non-Adaptive and Adaptive tests

Based on Regressed Rasch Difficulties

\begin{tabular}{ccccc} 
Number of Items & Minutes & Non-Adaptive & Adaptive & Gain \\
\hline 16 & 9.1 & .795 & .836 & .041 \\
24 & 11.8 & .858 & .887 & .029 \\
32 & 14.6 & .892 & .915 & .023 \\
\hline
\end{tabular}


Table 4

Reliabilities of Non-Adaptive and Adaptive tests

Based on Regressed 2-PL Parameters

\begin{tabular}{ccccc} 
Number of Items & Minutes & Non-Adaptive & Adaptive & Gain \\
\hline 16 & 9.1 & .800 & .847 & .047 \\
24 & 11.8 & .862 & .894 & .032 \\
32 & 14.6 & .895 & .919 & .024 \\
\hline
\end{tabular}

Another simulation with 155,000 uniformly distributed examinees was used to compute the information functions for adaptive and non-adaptive tests. The results for 24item tests are shown in Figure 4. Two curves are shown for the same non-adaptive test, based on the Rasch estimated parameters (dashed lines) and on the 2-PL regression estimates (solid lines). The information functions of the adaptive tests are shown with their confidence bands uniformly higher than the information functions for the non-adaptive test. Although the gain in reliability from adaptive testing is small, there is a substantial improvement in the precision of measurement for low and high-ability examinees.

The information functions for the Rasch tests and the 2-PL tests cross at about -.25 on the $\theta$ scale. This is because the constant $a$ parameter used in the Rasch model is greater than the regression estimates of $a$ used by the 2-PL model for 5-digit and 6-digit items.

Insert Figure 4 about here.

A second simulation study was conducted in which the parameters used to generate the items were different from those used by the adaptive testing algorithm to select items and score the test. The parameters estimated by MULTILOG for the two-parameter model were assumed to be the "true" values that were used to generate the simulated examinees' item responses. The parameters used for adaptive testing were, as before, the estimates that fell on the regression lines. Table 5 shows the effect on reliability. While the relabilities are lower than in the first simulation (Tables 3 and 4), the adaptive test 


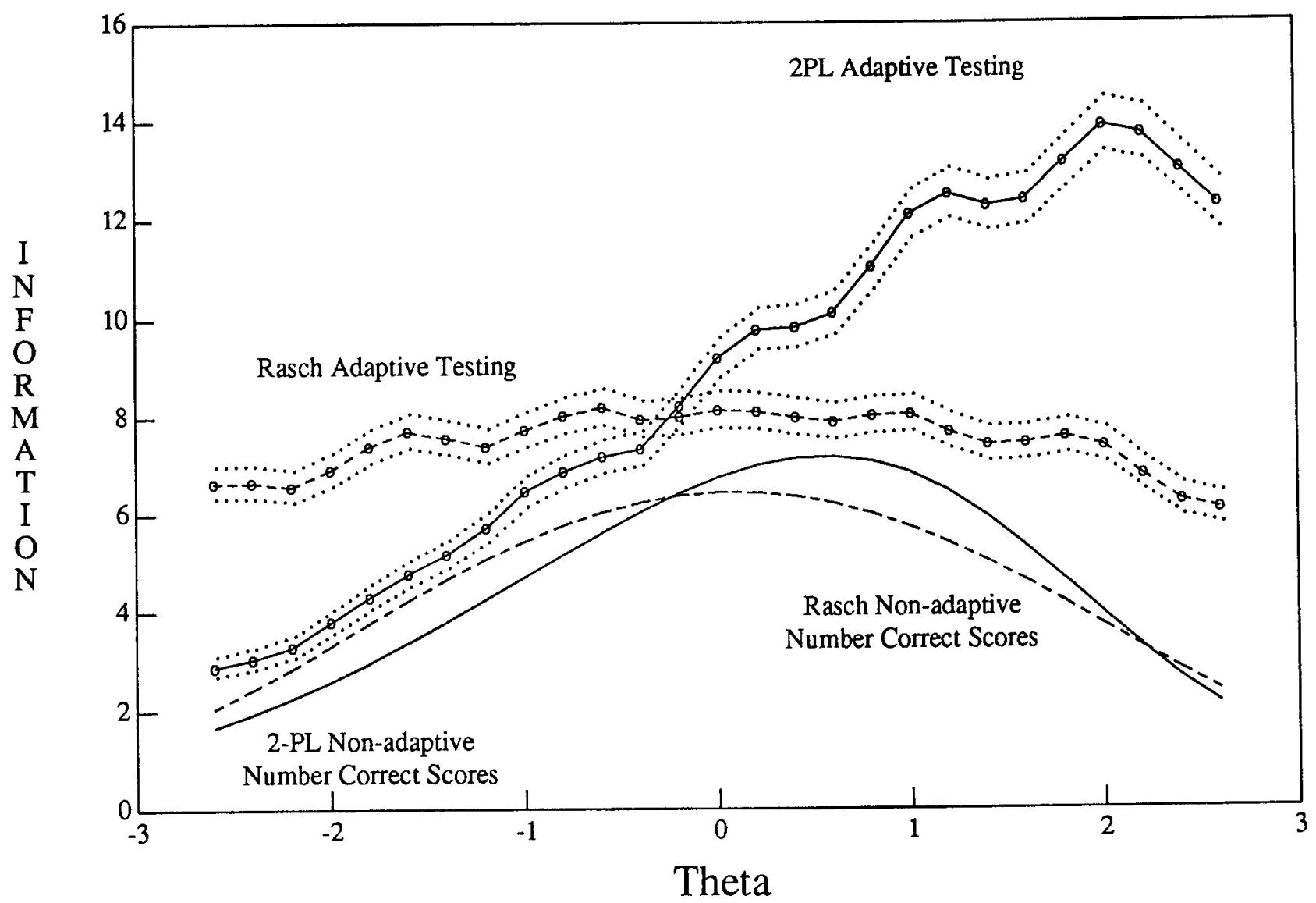

Figure 4. Information Functions of Non-adaptive Testing, Linearized Rasch Adaptive Testing, and Linearized 2PL Adaptive Testing using "Errorless" Parameters for 24-item Digit Span Tests. (Circles show ability levels where adaptive testing was simulated; dotted lines show confidence intervals) 
based on the 2-PL regression model is clearly more reliable than the other tests.

Table 5

Reliabilities of Non-Adaptive and Adaptive tests

Simulated from MULTILOG 2-PL Parameters

\begin{tabular}{ccccc} 
& & & Linear & Linear \\
Number of Items & Minutes & Non-Adaptive & 2-PL \\
\hline 16 & 9.1 & .776 & .802 & .823 \\
24 & 11.8 & .844 & .864 & .877 \\
32 & 14.6 & .881 & .897 & .906 \\
\hline
\end{tabular}

Figure 5 shows the information functions for the same three tests with 24 items each. The linearized Rasch and 2-PL curves cross at ability -0.75 , so that the Rasch adaptive test is more precise at the lower ability levels. This is undoubtedly due to the poor fit of the 2-PL regression lines to the parameters for 5-and 6-digit items.

Insert Figure 5 about here.

\section{ITEM GENERATION}

An item generation program was developed for the purpose of generating items of uniform difficulty for a given length. ${ }^{\dagger}$ Starting with a uniform pseudo-random number generator, digits from 0 to 9 were sampled without replacement, so that no repetitions or duplications were allowed. No item was allowed to begin or end with 0 or 1 . No two consecutive digits were allowed to differ by +1 or -1 . Items were discarded if their first three digits were identical to the first three digits of any other of the preceding 64 items generated. Finally, for each item, an "associational profile" was computed in the form of a 0,1 matrix with a 1 in row $i$, column $j$ if digit $i$ was immediately followed by $j$ in the item. A weighted moving average of associational profiles over the preceding items was formed, and a new item was discarded if its associational profile was too similar to the average associational profile of the preceding items.

The "seed" for the pseudo-random number generator was determined either by (a) setting it to a constant to generate a large sample of items for research, or (b) combining the ID number of the examinee with the date and time of the examination in order to generate a unique test for that examinee and occasion.

$\lceil$ The FORTRAN source code implementing this procedure is available on request. Address inquiries to John H. Wolfe, Navy Personnel Research and Development Center, San Diego, CA 92152-6800. 


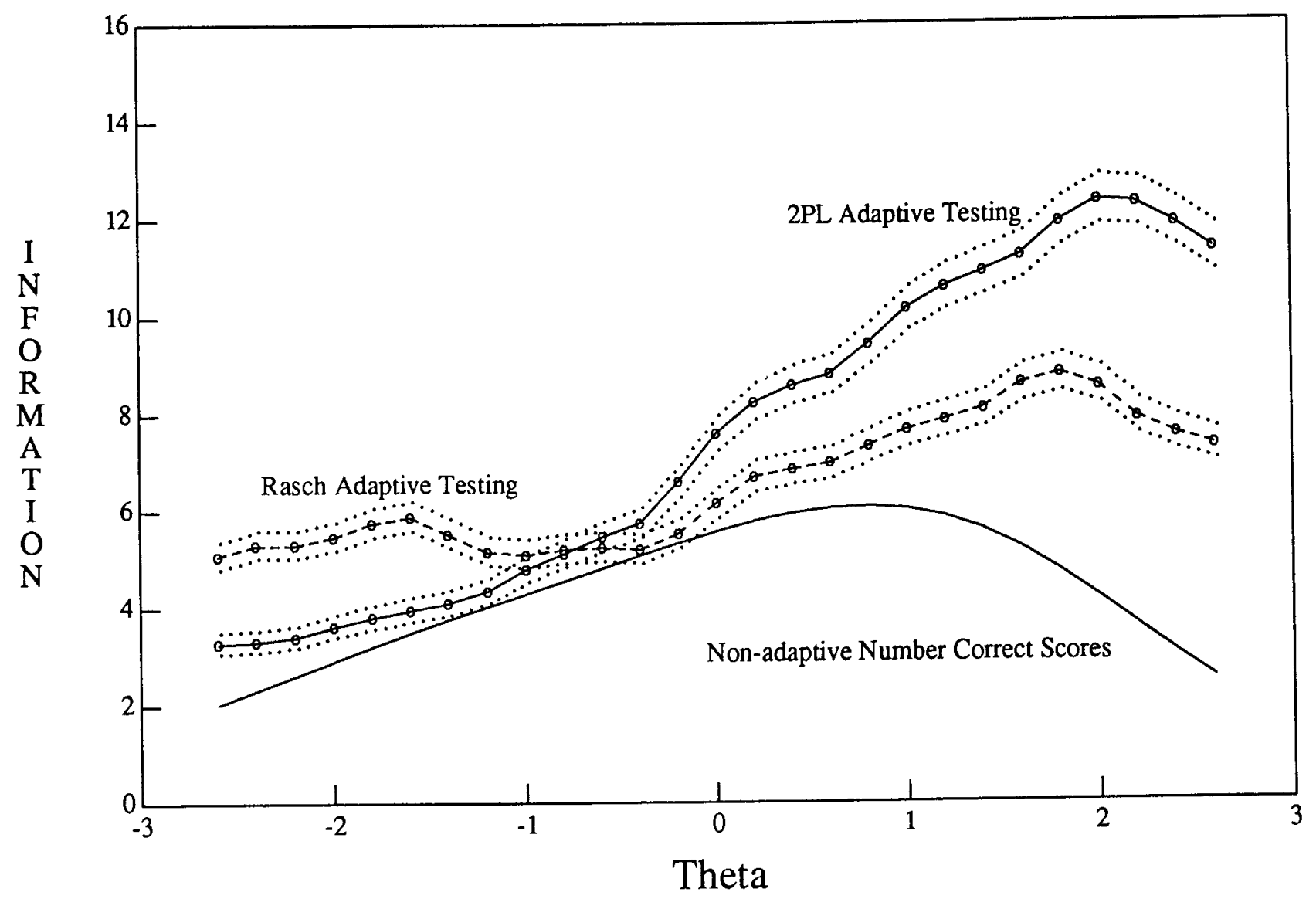

Figure. 5 Information Functions of Non-adaptive Testing, Linearized Rasch Adaptive Testing, and Linearized 2PL Adaptive Testing using True 2PL Parameters for 24-item Digit Span Tests. (Circles show ability levels where adaptive testing was simulated; dotted lines show confidence intervals) 


\section{GENERATIVE ADAPTIVE TESTING}

In a generative adaptive test, the item parameters for each generated item are estimated from the characteristics of the item. In this case, we constructed a generative digit span test using the Rasch difficulties estimated from the regression line determined in the model above. The items ranged in length from 4 to 10 digits. Items were constructed using the item generation procedure described above. After each item. Owen's bayesian ability estimate was computed (Owen, 1975), and the difficulty of the next item generated for administration was closer to the estimated examinee ability than any other item would be. The test length was 24 items. 


\section{REFERENCES}

Beaumont, J.G. (1985). The effect of microcomputer presentation and response medium on digit span performance. International Journal of Man Machine Studies, 22, 11-18.

Dempster, F. N. (1981). Memory span: sources of individual and developmental differences. Psychological Bulletin, 89, 63-100.

Mishra, S. P., Ferguson, B. A., \& King, P. V. (1985). Research with the Wechsler Digit Span subtest: Implications for assessment. School Psychology Review, 14, 37-47.

Slotnick, R. S.\& Jeger, A. M. (1985, August). Instructional Technology: Demonstration of Original Software for Teaching Introductory Psychology. Paper presented at the Annual Convention of the American Psychological Association, Los Angeles, CA.

Webster, J.S., Gouvier, W. D., \& Gross, R.T. (1983). A portable computerized technique for assessing visual digit span. Behavioral Engineering, 8, 98-101.

Wilson, S. (1982). Automated psychological testing for the severely physically handicapped. International Journal of Man Machine Studies, 17, 291-296.

Wilson, S. (1987). The development of an automated test of immediate memory and its evaluation on severely physically disabled adults. Applied Psychology, 36, 311-327. 


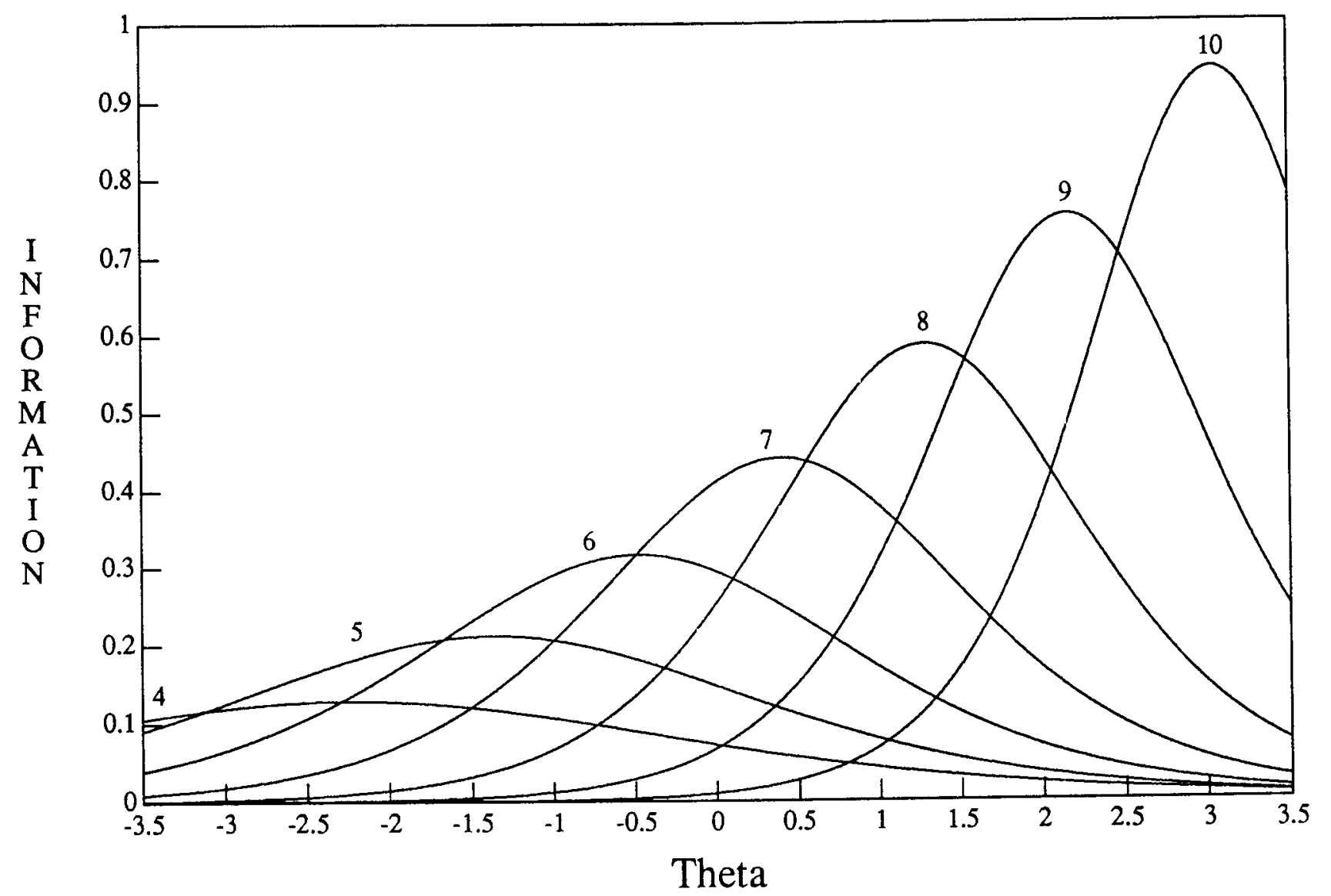

Figure. \# Information Functions of Digit Span Items from Regressed 2-PL Parameters. 